Pacific Journal of Mathematics

LIES WHOSE ANNIIATTORS ARE DIRECT 


\section{MODULES WHOSE ANNIHILATORS ARE DIRECT SUMMANDS}

\section{Charles W. Curtis}

Introduction. Let $B$ be a ring with an identity element, and let $M$ be a right $B$-module. The set of all elements $b$ in $B$ such that $M b=(0)$ is called the annihilator of $M$, and will be denoted by $(0: M)$. It is a natural question to ask under what circumstances the ideal $(0: M)$ is a direct summand of $B$. If $B$ is a semi-simple ring with minimum condition, for example, then every ideal is a direct summand, and there is no problem. We shall be concerned with a ring $B$, not assumed to be semi-simple, which is a crossed product $A(G, H, \rho)$ of a finite group $G$ and a division ring $\Delta$, with factor set $\rho$. In particular, $B$ may be the group algebra of a finite group with coefficients in a field. The purpose of this note is to obtain necessary and sufficient conditions on the structure of the module $M$ in order that its annihilator $(0: M)$ be a direct summand of $B$.

Our interest in the problem stems chiefly from the fact that the the modules whose annihilators are direct summands turn out to be precisely the modules for which the pairing defined in $\S 2$ of [1] is regular in the sense of $[1, p .281]$. The main results of [1], given in $\S 5$ and $\S 6$, are based upon the assumption that the pairing is regular, and establish a connection between the structure of the module $M$ relative to the set of $B$-endomorphisms of $M$ and the structure of a certain ideal in $B$, called the nucleus of $M$, which is the uniquely determined complementary ideal to $(0: M)$ when $(0: M)$ is a direct summand.

2. Familiarity with crossed products and their connection with projective representations of finite groups is assumed (see $[1, \S 2]$ ). In this section we recall some of the properties of a crossed product, and introduce, in a more general, and at the same time, much simpler fashion, the pairing defined in a special case by formula (7) of [1]. Let $G=$ $\{1, s, t, \cdots\}$ be a finite group, $\Delta$ a division ring and $B=\Delta(G, H, \rho)$ a crossed product of $G$ and $\Delta$ with correspondence $s \rightarrow \bar{s}=s^{I I}$ from $G$ to the group of automorphisms of $\Delta$, and factor set $\left\{\rho_{s, t}\right\}$. There exist elements $\left\{b_{1}, b_{s}, \cdots\right\}$ in $B$ in one-to-one correspondence with the elements of $G$, such that every element of $B$ can be expressed uniquely in the form $\sum b_{s} \xi_{s}$, with coefficients $\xi_{s}$ in $\Delta$. The multiplication in $B$ is determined by the equations

$$
b_{s} b_{t}=b_{s t} \rho_{s, t} ; \xi b_{s}=b_{s} \xi^{\bar{s}},
$$

This paper was originally accepted by the Trans. Amer. Math. Soc. Presented to the Society April 20, 1957; received by the editors of the Trans. Amer. Math. Soc., August 12, 1957. 
The fact that $B$ is an associative ring implies that the factor set $\left\{\rho_{s, t}\right\}$ satisfies the equations

$$
\rho_{s, t u} \rho_{t, u}=\rho_{s t, u} \rho_{s, t}^{\bar{u}},
$$

for all $s, t, u$ in $G$. We shall assume that the factor set $\rho$ is normalized so that $\rho_{1, t}=\rho_{t, 1}=1$ for all $t$ in $G$; then $b_{1}$ is the identity element in $B$.

The additive group of $B$ is a right vector space over $\Delta$ which we shall denote by $B^{(r)}$, if we define scalar multiplication by $\xi \in \Delta$ by means of the right multiplication $\xi_{r}: x \rightarrow x \xi$. Similarly the additive group of $B$ can be regarded as a left vector space $B^{(l)}$ over $\Delta$. The elements $b_{1}, b_{s}, \cdots$ form bases for both of these spaces. Because both spaces are finite dimensional, $B$ satisfies both chain conditions for left and right ideals.

The mapping $\lambda: \sum b_{s} \xi_{s} \rightarrow \xi_{1}$ is a linear function on both vector spaces $B^{(r)}$ and $B^{(l)}$ whose kernel contains no left or right ideal different from zero. Therefore the mapping $\Lambda: \Lambda(a, b)=\lambda(a b)$ is a non-degenerate bilinear form on $B^{(l)} \times B^{(r)} \rightarrow \Delta$. Using the bilinear form $\Lambda$ it is easy to verify (cf. [1, p. 279]) that $B$ is a quasi-Frobenius ring, that is, $B$ satisfies the minimum condition, and every right ideal in $B$ is the right annihilator of its left annihilator, and similarly for left ideals.

A right $B$-module - $^{1}$ is a fortiori a right vector space over $\Delta$ since $\Delta \subset B$. For each $s$ in $G$, the mapping $T_{s}: x \rightarrow x b_{s}$ is a semi-linear transformation belonging to the automorphism $\bar{s}$ in this vector space. The correspondence $s \rightarrow T_{s}$ defines a projective representation of $G$. Each transformation $T_{s}$ has an inverse $T_{s}^{-1}$ which is a semilinear transformation with automorphism $\bar{s}^{-1}$. Let $M^{\prime}$ be any left vector space over $\Delta$ which is paired with $M$ to $\Delta$ by a non-degenerate bilinear form $f$. Let us assume also that the semi-linear transformations $T_{s}$ all possess transposes $T_{s}^{*}$ with respect to the form $f$, such that

$$
f\left(\psi, x T_{s}\right)=f\left(T_{s}^{*} \psi, x\right)^{\bar{s}},
$$

for all $x \in M, \psi \in M^{\prime}$. If we define $\left(\sum b_{s} \xi_{s}\right) \psi=\sum T_{s}^{*}\left(\xi_{s} \psi\right)$, then $M^{\prime}$ becomes a left $B$-module (see [1, p. 274]). When these conditions are satisfied, we shall call the system $\left(M^{\prime}, M, f\right)$ a pair of dual $B$-modules.

Lemma 1. Let $\left(M^{\prime}, M, f\right)$ be a pair of dual B-modules. Then the function

$$
\tau_{f}(\psi, x)=\sum_{s \in G} f\left(\psi, x T_{s}\right) b_{s}^{-1}
$$

is a non-degenerate B-bilinear function on $M^{\prime} \times M \rightarrow B$ (cf. [1, Proposition 1]).

1 We shall assume that the identity element of $B$ acts as the identity operator on all modules we shall consider. 
Proof. For any $u \in G$ we have

$$
\begin{aligned}
b_{u}^{-1} \tau_{f}\left(T_{u}^{*} \psi, x\right) & =\sum_{s \in G} b_{u}^{-1} f\left(T_{u}^{*} \psi, x T_{s}\right) b_{s}^{-1} \\
& =\sum_{s \in G} f\left(\psi, x T_{s} T_{u}\right) b_{u}^{-1} b_{s}^{-1}=\tau_{f}(\psi, x)
\end{aligned}
$$

by (1) and (3). Similarly, for all $u$,

$$
\tau_{f}\left(\psi, x T_{u}\right) b_{u}^{-1}=\tau_{f}(\psi, x) .
$$

Since the function $\tau_{f}$ is obviously bilinear as far as $\Delta$ is concerned, these calculations establish that for all $b \in B$,

$$
b \tau_{f}(\psi, x)=\tau_{f}(b \psi, x) \text { and } \tau_{f}(\psi, x b)=\tau_{f}(\psi, x) b .
$$

The non-degeneracy of $\tau_{f}$ follows at once from the non-degeneracy of $f$.

To each right $B$-module $M$ corresponds a two-sided ideal $B_{M}$ in $B$, defined as follows. Find a left $B$-module $M^{\prime}$ which is paired with $M$ to $\Delta$ by a non-degenerate bilinear form $f$ such that $\left(M^{\prime}, M, f\right)$ is a pair of dual $B$-modules (for example, the space $M^{\prime}$ of all linear functions on $M$ can be used). Then by Lemma 1 , the set $B_{M}$ consisting of all finite sums $\sum \tau_{f}\left(\psi_{i}, x_{i}\right), \psi_{i} \in M^{\prime}, x_{i} \in M$, is a two-sided ideal in $B$. We shall call $B_{M}$ the nucleus of $M$. We leave it to to the reader to verify that, as our notation indicates, $B_{M}$ is independent of the choice of $M^{\prime}$ and $f$.

We now define a right $B$-module $M$ to be a regular module if $B_{M}$ contains an element $\varepsilon$ such that $\varepsilon b=b \varepsilon=b$ for all $b \in B_{M}$. We remark that the statement that $M$ is a regular module is equivalent to the statement, in the terminology of [1], that $\left(M^{\prime}, M, \tau_{f}\right)$ is a regular pairing (see [1, p. 281]).

3. This section contains some lemmas on regular modules. We remark first that if $M_{1}$ and $M_{2}$ are isomorphic $B$-modules, then $B_{M_{1}}=B_{M_{2}}$, and hence regularity is preserved under isomorphism.

Lemma 2. The nucleus $B_{M}$ and the annihilator $(0: M)$ of a regular module $M$ are two-sided ideals in $B$ generated by central idempotents, and $B=(0: M) \oplus B_{M} .^{2}$

Proof. Let $\left(M^{\prime}, M, f\right)$ be a pair of dual $B$-modules, where $M$ is the given regular module. By Theorem 1, p. 282, of [1], we have $B_{r}=$ $\left(B_{M}\right)_{r}$, and consequently $B=B_{M}+(0: M)$. Let $\varepsilon=\sum \tau_{f}\left(\psi_{i}, x_{i}\right)$ be the identity element in $B_{M}$. Then $a \in B_{M} \cap(0: M)$ implies $a=\varepsilon a=$ $\sum \tau_{f}\left(\psi_{i}^{\prime} x_{i} a\right)=0$, and the sum is direct. We have $\varepsilon^{\prime}=1-\varepsilon \in(0: M)$, and because $B_{M}$ and $(0: M)$ are ideals whose intersection is zero, $\varepsilon$ and $\varepsilon^{\prime}$ are orthogonal central idempotents which generate $B_{M}$ and $(0: M)$

2 We take this opportunity to correct an error in [1]. The assertion made in example (c) in $\S 11$, p. 291 , of [1] that $0 \neq(0: M) \subset B_{M}$ for a certain regular module $M$ is false and the assertion (c) should be deleted from [1]. 
respectively.

LEMMA 3. Let $M$ be a right $B$-module such that $M=M_{1} \oplus M_{2}$, where $M_{1}$ and $M_{2}$ are submodules. Let $M^{\prime}$ be the space of all linear functions on $M$, paired with $M$ to $\Delta$ by the function $f$ defined by $f(\psi, x)=\psi(x)$, $\psi \in M^{\prime}, x \in M$. Then $\left(M^{\prime}, M, f\right)$ is a pair of dual B-modules, Let $M_{!}^{\perp}$ and $M_{2}^{\perp}$ be the subspaces of $M^{\prime}$ which annihilate $M_{1}$ and $M_{2}$ respectively. Then $M^{\prime}=M_{1}^{\perp} \oplus M_{2}^{\perp}$; the restrictions $f_{1}$ and $f_{2}$ of $f$ to $M^{\perp} \times M_{1}$ and $M_{1}^{\perp} \times M_{2}$, respectively, are non-degenerate; and $\left(M_{2}^{\perp}, M_{1}, f_{1}\right)$, and $\left(M_{1}^{\perp}, M_{2}, f_{2}\right)$ are pairs of dual $B$-modules.

Proof. The semi-linear transformations $T_{s}$ all possess transposes $T_{s}^{*}$ relative to the form $f$, such that formula (3) holds, and consequently $\left(M^{\prime}, M, f\right)$ is a pair of dual $B$-modules. The sets $M_{1}^{\perp}$ and $M_{2}^{\perp}$ are subspaces of $M^{\prime}$ such that $M_{1}^{\perp} \cap M_{2}^{\perp}=(0)$. If $\psi \in M^{\prime}$, then $\psi \mid M_{1}=\psi_{1}$ is a linear function on $M_{1}$, which can be extended to a linear function $\psi_{1}$ in $M^{\prime}$ by setting $\psi_{1} \mid M_{2}=0$. Similarly we define $\psi_{2}$. Then $\psi=\psi_{1}+\psi_{2}$, and we have proved that $M^{\prime}=M_{2}^{\perp} \oplus M_{1}^{\perp}$. The restrictions $f_{1}$ and $f_{2}$ defined in the statement of the lemma are clearly non-degenerate. Finally, since $M_{1}$ and $M_{2}$ are $B$-submodules, it follows from (3) that $T_{s}^{*}\left(M_{i}^{\frac{1}{i}}\right) \subseteq M_{i}^{\frac{1}{i}}$, $i=1,2$, and hence $T_{s} \mid M_{i}$ has the transpose $T_{s}^{*} \mid M_{2-i}^{\perp}, i=1,2$, and the proof is complete.

Lemma 4. Let $M=M_{1} \oplus M_{2}$, where $M_{1}$ and $M_{2}$ are $B$-submodules of M. Then $B_{M}=B_{M_{1}}+B_{M_{2}}$.

Proof. Let $M^{\prime}$ be the space of all linear functions on $M$, and define $f, f_{1}, f_{2}$ as in Lemma 3 . Let $\tau_{f}, \tau_{f_{1}}, \tau_{f_{2}}$ be the corresponding functions defined by (4). For $x \in M_{1}, \psi \in M_{2}^{\perp}$, we have $\tau_{f_{1}}(\psi, x)=\tau_{f}(\psi, x)$ and $B_{M_{1}} \subseteq B_{M}$. Similarly $B_{M_{2}} \subseteq B_{M}$. Now let $x \in M$, and write $x=x_{1}+x_{2}$, $x_{i} \in M_{i}$; and let $\psi \in M^{\prime}, \psi=\psi_{1}+\psi_{2}, \psi_{1} \in M_{2}^{\perp}, \psi_{2} \in M_{1}^{\perp}$. Then since $M_{1}$ and $M_{2}$ are submodules we have

$$
\begin{aligned}
\tau_{f}(\psi, x) & =\sum f\left(\psi_{1}+\psi_{2},\left(x_{1}+x_{2}\right) b_{s}\right) b_{s}^{-1} \\
& =\sum f_{1}\left(\psi_{1}, x_{1} b_{s}\right) b_{s}^{-1}+\sum f_{2}\left(\psi_{2}, x_{2} b_{s}\right) b_{s}^{-1} \\
& =\tau_{f_{1}}\left(\psi_{1}, x_{1}\right)+\tau_{f_{2}}\left(\psi_{2}, x_{2}\right),
\end{aligned}
$$

and the lemma is proved.

Lemma 5. Let $M=M_{1} \oplus M_{2}$ where $M_{1}$ and $M_{2}$ are regular $B$-modules. Then $M$ is a regular $B$-module.

Proof. By Lemma 4, $B_{M}=B_{M_{1}}+B_{M_{2}}$. By Lemma 2, we have $B_{M i}=\varepsilon_{i} B$ where $\varepsilon_{i}$ is a central idempotent, $i=1$, 2. Then $\varepsilon=\varepsilon_{1}+\varepsilon_{2}-\varepsilon_{1} \varepsilon_{2} \in B_{M}$, and $b \varepsilon=\varepsilon b=b$ for all $b \in B_{M}$, proving our assertion.

Lemma 6. Let $e$ be an idempotent in $B$. Then $(B e, e B, A)$ is a pair of dual $B$-modules.

Proof. We recall from $\S 2$ that $\Lambda$ is a non-degenerate bilinear form 
on $B^{(l)} \times B^{(r)} \rightarrow \Delta$. The restriction of $A$ to $B e \times e B$ is also non-degenerate (see [1], p. 279). It remains to verify that for all $c, d$ in $B$,

$$
\Lambda\left(c, d b_{s}\right)=\Lambda\left(b_{s} c, d\right)^{\bar{s}} \text {. }
$$

For this it is sufficient to prove that if $a=\sum \xi_{u} b_{u}=\sum b_{u} \xi_{u}^{\bar{u}}$, then $\lambda\left(a b_{s}\right)=$ $\lambda\left(b_{s} a\right)^{\bar{s}}$ for all $s \in G$. We have $\lambda\left(a b_{s}\right)=\xi_{s^{-1}} \rho_{s^{-1}, s}$, while

$$
\lambda\left(b_{s} a\right)^{\bar{s}}=\rho_{s, s^{-1}}^{\bar{s} \xi_{s}^{s-1}-\bar{s}}=\rho_{s, s^{-1}}^{\bar{s}} \rho_{s}^{-1}, \xi_{s} \xi_{s}^{-1} \rho_{s}^{-1}, s
$$

by formula (2) of [1], and by (2) above we have

$$
\rho_{1, s} \rho_{s, s}^{\bar{s}}{ }^{-1}=\rho_{s, 1} \rho_{s}^{-1, s},
$$

and the formula (5) is proved.

4. Now we shall formulate and prove our main result. Because $B$ satisfies the minimum condition, $B=B_{1} \oplus \cdots \oplus B_{r}$, where the $B_{i}$ are uniquely determined indecomposable two-sided ideals, called the block ideals ${ }^{3}$ of $B$. If we write $1=\varepsilon_{1}+\cdots+\varepsilon_{r}, \varepsilon_{i} \in B_{i}$, then the $\varepsilon_{i}$ are mutually orthogonal idempotents belonging to the center of $B$, and $\varepsilon_{i}$ is the identity element in the block ideal to which it belongs. For any right $B$-module $M, M \varepsilon_{i}$ is a submodule of $M$, and $M$ is the direct sum of the modules $M \varepsilon_{i}$. These submodules are called the block components of $M$; the block component $M \varepsilon_{i}$ can also be described as the set of elements of $M$ which are left fixed by $\varepsilon_{i}$. The block components of $(B,+)$, where $(B,+)$ is viewed as a right $B$-module in the obvious way, are the block ideals $B \varepsilon_{i}$. Each block component $B \varepsilon_{i}$ of $B$ can be expressed as a direct sum of the indecomposable right ideals $e_{k} B, e_{k}^{2}=e_{k}$, which belong to the block. It is known that two indecomposable right ideals $e B$ and $e^{\prime} B$ belonging to distinct blocks have no isomorphic composition factors. The direct sum of a full set of non-isomorphic indecomposable right ideals $e_{k} B$ belonging to the $i$ th block component $B \varepsilon_{i}$ of $B$, or any right $B$-module isomorphic to this module, is called a reduced block component of $B$.

Our theorem is stated as follows.

Theorem. Let $M$ be a right $B$-module with annihilator $(0: M)$. The following statements are equivalent.

(A) $(0: M)$ is a direct summand of $B$;

(B) every non-zero block component $M \varepsilon_{i}$ of $M$ contains the $i$ th reduced block component of $B$ as a direct summand;

(C) $M$ is a regular module.

Proof. The implication $(\mathrm{C}) \rightarrow(\mathrm{A})$ is the content of Lemma 2. We prove next that $(A) \rightarrow(B)$. Let $B^{\prime}$ be a two sided ideal in $B$ such that

3 For the concepts of block ideals and block components see [3], and the references given there. 
$B=B^{\prime} \oplus(0: M)$. By the uniqueness of the decomposition of $B$ into block ideals, $B^{\prime}$ is a direct sum of certain of the block ideals $B \varepsilon_{i}$. Let $M \varepsilon_{i}$ be a non-zero block component of $M$; then $B \varepsilon_{i} \subseteq B^{\prime}$, and $M \varepsilon_{i}$ is a faithful $B \varepsilon_{i}$ module. Let $e B$ be an indecomposable right ideal belonging to the $i$ th block. By Proposition 4 of [1], eB contains a unique minimal right ideal $N \neq(0)$. There exists an element $x \in M$ such that $x N \neq(0)$. It follows that $u \rightarrow x u$ is a $B$-isomorphism of $e B$ onto the submodule $P=x e B$ of $M \varepsilon_{i}$. We shall prove that there exists a submodule $Q$ of $M \varepsilon_{i}$ such that $M \varepsilon_{i}=Q \oplus P$. Let $M^{\prime}$ be the set of all linear functions on $M \varepsilon_{i}$, paired with $M \varepsilon_{i}$ to $\Delta$ by the non-degenerate bilinear form $f$, so that $\left(M^{\prime}, M \varepsilon_{i}, f\right)$ is a pair of dual $B$-modules. Let $P^{\perp}$ be the submodule of $M^{\prime}$ consisting of all elements $\psi \in M^{\prime}$ such that $f(\psi, P)=(0)$. Then $\left(M^{\prime} \mid P^{\perp}, P, \bar{f}\right)$ is a pair of dual $B$-modules, where $\bar{f}$ is the induced mapping on $M^{\prime} \mid P^{\perp} \times P$. On the other hand, by Lemma $6,(B e, e B, \Lambda)$ is a pair of dual $B$-modules. Using the fact that $e B$ is a finite dimensional space, it is easily verified that $B e$ and $M^{\prime} \mid P^{\perp}$ are isomorphic left $B$ modules. By Theorem 1 of [2], $B e$ is an $\left(M_{0}\right)$-module, and consequently there exists a $B$-submodule $Q^{\prime}$ of $M^{\prime}$ such that $M^{\prime}=P^{\perp} \oplus Q^{\prime}$. Let $Q=\left\{x \mid x \in M \varepsilon_{i}, f\left(Q^{\prime}, x\right)=(0)\right\}$. Then $Q$ is a submodule such that $P \cap Q=(0)$. Moreover

$$
M=\left(P^{\perp} \cap Q^{\prime}\right)^{\perp}=P^{\perp}+\left(Q^{\prime}\right)^{\perp}=P+Q,
$$

since $P$ is finite dimensional and $Q=\left(Q^{\prime}\right)^{\perp}$.

The proof that $M \varepsilon_{i}$ contains the reduced block component of $B \varepsilon_{i}$ as a direct summand is now proved by induction. Let $M \varepsilon_{i}=R \oplus S$, where $R$ is isomorphic to a direct sum of a finite number of non-isomorphic indecomposable right ideals belonging to the $i$ th block, and let $e B$ be an indecomposable right ideal in $B \varepsilon_{i}$ not isomorphic to any of the direct summands of $R$. Let $N$ be the unique minimal subideal of $e B$. If $R N \neq(0)$, then by the previous argument $R$ contains a direct summand isomorphic to $e B$, which contradicts the Krull-Schmidt theorem. Thus $R N=(0)$, and $S N \neq(0)$, so that $S$ contains a direct summand isomorphic to $e B$. This completes the proof of the induction step, and the implication $(\mathrm{A}) \rightarrow(\mathrm{B})$ is established.

Finally we prove that $(\mathrm{B}) \rightarrow(\mathrm{C})$. By Lemma 5 , it is sufficient to prove that each block component $M \varepsilon_{i}$ of $M$ is a regular module, and for this it is sufficient to show that $\varepsilon_{i} \in B_{M i}$ whenever $M \varepsilon_{i} \neq(0)$. Let us consider a non-zero component $M \varepsilon_{i}$. Let $e_{1} B, \cdots, e_{s} B$ be a full set of non-isomorphic indecomposable right ideals belonging to the $i^{\text {th }}$ block. For each $j, 1 \leqq j \leqq s$, there exists a $B$-direct summand $P_{j}$ of $M \varepsilon_{i}$ such that $P_{j} \cong e_{j} B$. By Lemma $4, B_{P_{j}}=B_{e_{j} B} \cong B_{M \varepsilon i}$. We prove that $e_{j} \in B_{e_{j} B}$. By Lemma $6,\left(B e_{j}, e_{j} B, A\right)$ is a pair of dual $B$-modules. We assert that

$$
e_{j}=\tau_{\Lambda}\left(e_{j}, e_{j}\right) \text {. }
$$


In fact, $\tau_{\Lambda}\left(e_{j}, e_{j}\right)=\sum \Lambda\left(e_{j}, e_{j} b_{s}\right) b_{s}^{-1}$, and if $e_{j}=\sum \xi_{u} b_{u}$, then

$$
\Lambda\left(e_{j}, e_{j} b_{s}\right)=\lambda\left(e_{j} b_{s}\right)=\xi_{s}-1 \rho_{s^{-1}, s}
$$

while from $b_{s}-1 b_{s}=b_{1} \rho_{s^{-1}, s}$ we have $b_{s}^{-1}=\rho_{s^{-1}, s}^{-1} b_{s^{-1}}$. From these remarks (6) follows.

We have shown that $e_{j} \in B_{M \varepsilon i}$. Since $\varepsilon_{i}$ is a sum of idempotents $e$ such that $e B$ is isomorphic to one of the ideals $e_{j} B, 1 \leqq j \leqq s$, we have $\varepsilon_{i} \in B_{M \varepsilon i}$, and $M \varepsilon_{i}$ is a regular module. This completes the proof of the theorem.

\section{REFERENCES}

1. C. W. Curtis, Commuting rings of endomorphisms, Can. J. Math., 8 (1956), 271-292.

2. H. Nagao, and T. Nakayama, On ihe structure of $\left(\boldsymbol{M}_{0}\right)$ and $\left(\boldsymbol{M}_{u}\right)$ modules, Math. Zeit., 59 (1953), 164-170.

3. C. Nesbitt, and R. Thrall, Some ring theorems with applications to modular representations, Ann. of Math,. 47 (1946), 551-567.

UNIVERSITY OF WISCONSIN 



\section{PACIFIC JOURNAL OF MATHEMATICS}

\section{EDITORS}

\section{David Gilbarg}

Stanford University

Stanford, California

\section{R. A. Beaumont}

University of Washington

Seattle 5, Washington

\author{
A. L. Whiteman
}

University of Southern California Los Angeles 7, California

E. G. Straus

University of California

Los Angeles 24, California

\section{ASSOCIATE EDITORS}

\author{
E. F. BECKENBACH \\ C. E. BURGESS \\ M. HALL \\ E. HEWITT
}
A. HORN
V. GANAPATHY IYER
R. D. JAMES
M. S. KNEBELMAN
L. NACHBIN
I. NIVEN
T. G. OSTROM
H. L. ROYDEN

M. M. SCHIFFER

G. SZEKERES

F. WOLF

K. YOSIDA

\section{SUPPORTING INSTITUTIONS}

\author{
UNIVERSITY OF BRITISH COLUMBIA \\ CALIFORNIA INSTITUTE OF TECHNOLOGY \\ UNIVERSITY OF CALIFORNIA \\ MONTANA STATE UNIVERSITY \\ UNIVERSITY OF NEVADA \\ OREGON STATE COLLEGE \\ UNIVERSITY OF OREGON \\ OSAKA UNIVERSITY \\ UNIVERSITY OF SOUTHERN CALIFORNIA
}

\author{
STANFORD UNIVERSITY \\ UNIVERSITY OF TOKYO \\ UNIVERSITY OF UTAH \\ WASHINGTON STATE COLLEGE \\ UNIVERSITY OF WASHINGTON \\ * * * * \\ AMERICAN MATHEMATICAL SOCIETY \\ CALIFORNIA RESEARCH CORPORATION \\ HUGHES AIRCRAFT COMPANY \\ THE RAMO-WOOLDRIDGE CORPORATION
}

Mathematical papers intended for publication in the Pacific Journal of Mathematics should be typewritten (double spaced), and the author should keep a complete copy. Manuscripts may be sent to any one of the four editors. All other communications to the editors should be addressed to the managing editor, E. G. Straus at the University of California, Los Angeles 24, California.

50 reprints per author of each article are furnished free of charge; additional copies may be obtained at cost in multiples of 50 .

The Pacific Journal of Mathematics is published quarterly, in March, June, September, and December. The price per volume (4 numbers) is $\$ 12.00$; single issues, $\$ 3.50$. Back numbers are available. Special price to individual faculty members of supporting institutions and to individual members of the American Mathematical Society: $\$ 4.00$ per volume; single issues, $\$ 1.25$.

Subscriptions, orders for back numbers, and changes of address should be sent to Pacific Journal of Mathematics, 2120 Oxford Street, Berkeley 4, California.

Printed at Kokusai Bunken Insatsusha (International Academic Printing Co., Ltd.), No. 6, 2-chome, Fujimi-cho, Chiyoda-ku, Tokyo, Japan.

PUBLISHED BY PACIFIC JOURNAL OF MATHEMATICS, A NON-PROFIT CORPORATION

The Supporting Institutions listed above contribute to the cost of publication of this Journal, but they are not owners or publishers and have no responsibility for its content or policies. 


\section{Pacific Journal of Mathematics}

\section{Vol. 8, No. $4 \quad$ June, 1958}

Richard Arens, The maximal ideals of certain functions algebras ........ 641

Glen Earl Baxter, An operator identity ........................... 649

Robert James Blattner, Automorphic group representations ........... 665

Steve Jerome Bryant, Isomorphism order for Abelian groups ............ 679

Charles W. Curtis, Modules whose annihilators are direct summands...... 685

Wilbur Eugene Deskins, On the radical of a group algebra ............ 693

Jacob Feldman, Equivalence and perpendicularity of Gaussian

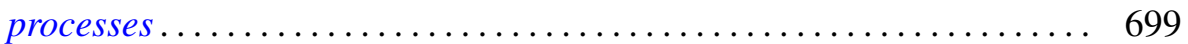

Marion K. Fort, Jr. and G. A. Hedlund, Minimal coverings of pairs by triples....................................... 709

I. S. Gál, On the theory of $(m, n)$-compact topological spaces ......... 721

David Gale and Oliver Gross, A note on polynomial and separable games........................................ 735

Frank Harary, On the number of bi-colored graphs ............... 743

Bruno Harris, Centralizers in Jordan algebras ................... 757

Martin Jurchescu, Modulus of a boundary component ............... 791

Hewitt Kenyon and A. P. Morse, Runs . . . . . . . . . . . . . . . . . . . . . . 811

Burnett C. Meyer and H. D. Sprinkle, Two nonseparable complete metric

spaces defined on $[0,1] \ldots \ldots \ldots \ldots \ldots \ldots \ldots \ldots \ldots \ldots \ldots . \ldots . \ldots . \ldots . \ldots 25$

M. S. Robertson, Cesàro partial sums of harmonic series expansions...... 829

John L. Selfridge and Ernst Gabor Straus, On the determination of numbers by their sums of a fixed order ........................ 847

Annette Sinclair, A general solution for a class of approximation

problems .................................

George Szekeres and Amnon Jakimovski, $(C, \infty)$ and $(H, \infty)$ methods of summation...................................... 867

Hale Trotter, Approximation of semi-groups of operators. ............. 887

L. E. Ward, A fixed point theorem for multi-valued functions ........... 921

Roy Edwin Wild, On the number of lattice points in $x^{t}+y^{t}=n^{t / 2} \ldots \ldots .929$ 